\title{
Effects of Total Flavonoids of Epimedium in Climacteric Model Rats
}

\author{
M.S. MIAO, W.Y. XIN, M. BAI, L. GUO, T. WANG \& H.L. LIU \\ Henan University of Traditional Chinese Medicine, Zhengzhou, Henan 450008, China
}

M.S. MIAO

Professor, Zhengzhou, Henan, China

\begin{abstract}
Objective: To investigate the effects of Total Flavonoids of Epimedium (TFE) in climacteric model rats. Method: Utilized castration method to make climacteric syndrome animal model and observed the effects of high middle and low doses of TFE(that is TFE-HD/TFE-MD/TFE-LD) on organ index, serum sex hormone, serum IL-2, BGP content in climacteric rats. Results: Compared with the blank group, the uterus index, spleen index and the level of serum E2, serum IL-2 and BGP levels of model group were significantly decreased $(\mathrm{P}<0.01)$, LH, FSH levels were significantly increased $(\mathrm{P}<0.01)$. Explained the incomplete removal of the ovaries rat model of climacteric appeared atrophy of spleen and uterus, sex hormone disordered and reduced the immunity and bone calcium.Compared with the model group, each treatment group could significantly increase the spleen index, TFE-MD could significantly improve the uterus index $(\mathrm{P}<0.05)$, TFEHD could significantly improve the uterus index $(\mathrm{P}<0.01)$. The TFE in each dosage group and soybean isoflavones (SI) group could significantly increase the level of serum E2(P<0.01), TFE-HD and SI could significantly decrease the serum LH level, TFE-HD could significantly decrease the levels of FSH $(\mathrm{P}<0.01)$. Gengnian'an capsule group and TFE-HD were significantly increase serum IL-2 level; SI significantly increased serum BGP level, other each group could significantly increase the level of serum BGP. Conclusion: TFE had significant effect on the treatment of climacteric syndrome.
\end{abstract}

KEYWORD: Total Flavonoids of Epimediu; Rats; Climacteric model

\section{INTRODUCTION}

Epimedium is Berberidaceae plant, pubescent Epimedium or Korean Epimedium's dried aerial parts. Its taste is xin, gan, wen, having effects on the livers, kidney. Its main functions are to invigorate kidney yang, strong muscles and bones, expel wind and dampness[1]. Climacteric syndrome is one of the common diseases of women, mainly because of ovarian function decline, caused endocrine disorders, decreased immunity and plant nerve disorder syndrome. TFE is extracted from Epimedium Flavonoids in stems and leaves, as the main effective components of Epimedium. Pharmacological studies show that it have a certain action on the immune system and nervous system diseases[2]. Modern research also shows that TFE can enhance the hypothalamus pituitary gonadal function, and has estrogen-like activity [3-4]. In this experiment, we used castration method to observe its effect on climacteric women, in order to screening of a new drug for climacteric syndrome, and provide a new idea for the treatment of menopausal disorders as well.

\section{MATERIALS}

\subsection{Instruments and reagents drugs}

FA (N) /JA (N) series electronic balance was purchased from Shanghai Minqiao Precision Instrument Co,Ltd. SN-695 B intelligent radioimmunoassay gamma survey meter was purchased from Shanghai Institute of nuclear research, ring instrument factory. In this study, TFE (identified content of 60\%) was provided by the Department of Chemistry, Henan College (batch number TY200080116). Gengnian'an capsules were purchased from Changchun English flat Pharmaceutical Co, Ltd (batch number Z20053759). SI tablets were purchased from Nanning Rich Leisching Biotechnology Co,Ltd (Southern Methodist food card characters (2006) 450101000389 ).

\subsection{Animals}

Kunming (KM) female rats in 160 200g weight (animal certificate No. 701022) was provided by the 
Medical Experimental Animal Center of Hebei province.

\subsection{Statistical analysis}

The SPSS 13.0 statistical software for Windows was used for data analysis. The measurement results were expressed as" mean \pm standard deviation $\left(\overline{\mathrm{x}}_{\mathrm{s}}\right)$ "). Comparison between groups was performed by the LSD method.

\section{METHODS}

80 female rats (weight, 160 200g) were randomly divided into seven groups. They were blank group, model group, Gengnian'an capsule group, soybean isoflavones (SI) group, TFE high dose group (TFEHD), TFE middle dose group (TFE-MD) and TFE low dose group (TFE-LD). Except the blank group, The rats were anesthetized after abdominal bit fixed, axillary line about $2 \mathrm{~cm}$ away from the spine at the last rib at shearing, cut the skin and back muscles, clip out the ovaries, the separation of cellulite, tubal ligation after removal of the left ovary all right side only removed $80 \%$ of ovarian[5]. After carefully kept, if infectioned then injection the penicillin, continuous three days. Five days after surgery then Vaginal smears in each rats, once a day for five continuous days, if the animals without estrus response, then the model was successed.

Administration: the drugs were suspended in 5\% CMC-Na solution. TFE-HD, TFE-MD, TFE-LD concentration of $10.5 \mathrm{mg} / \mathrm{ml}, 7 \mathrm{mg} / \mathrm{ml}, 3.5 \mathrm{mg} / \mathrm{ml}$, were administered $210 \mathrm{mg} / \mathrm{kg}, 140 \mathrm{mg} / \mathrm{kg}, 70 \mathrm{mg} / \mathrm{kg}$ respectively. Gengnian'an capsule concentration was $22.5 \mathrm{mg} / \mathrm{ml}$, according to $450 \mathrm{mg} / \mathrm{kg}$ administered; SI concentration was $4.25 \mathrm{mg} / \mathrm{ml}$, was administered $85 \mathrm{mg} / \mathrm{kg}$; model group and blank group were given the same volume of CMC-Na solution, one times a day for three continuous weeks.

\section{RESULTS}

\subsection{Effects of TFE on organ index in climacteric rats}

Compared with the blank group, the uterus index and spleen index of model group were significantly decreased $(P<0.01)$, explained that the incomplete removal of the ovaries rat model of menopause appear spleen and uterine atrophy. Compared with the model group, the spleen index of each group were significantly increased $(P<0.01)$; TFE-MD could significantly improve the uterus index $(P<0.05)$ and the uterus index of TFE-HD increased significantly $(P<0.01)$. These results are listed in Table 1.

Table 1 Effect of TFE on the spleen, thymus, uterus index in climacteric model rat $\left(\overline{\mathrm{X}}_{ \pm} \mathrm{s}\right)$

\begin{tabular}{|l|c|c|c|c|c|}
\hline Groups & $\mathrm{n}$ & $\begin{array}{c}\text { Dose } \\
(\mathrm{mg} / \mathrm{kg})\end{array}$ & $\begin{array}{c}\text { Thymus index } \\
(\mathrm{mg} / \mathrm{g})\end{array}$ & $\begin{array}{c}\text { Spleen index } \\
(\mathrm{mg} / \mathrm{g})\end{array}$ & $\begin{array}{c}\text { Uterus index } \\
(\mathrm{mg} / \mathrm{g})\end{array}$ \\
\hline Blank group & 10 & - & $1.729 \pm 0.494$ & $2.351 \pm 0.221$ & $1.787 \pm 0.624$ \\
\hline Model group & 10 & - & $1.431 \pm 0.199$ & $1.745 \pm 0.396^{\triangle \triangle}$ & $0.758 \pm 0.339^{\triangle \triangle}$ \\
\hline Gengnian'an capsule group & 10 & 450 & $1.771 \pm 0.505$ & $2.912 \pm 0.460 * *$ & $1.092 \pm 0.293$ \\
\hline SI group & 10 & 85 & $1.648 \pm 0.273$ & $2.932 \pm 0.632^{* *}$ & $0.835 \pm 0.239$ \\
\hline TFE-HD & 10 & 210 & $1.257 \pm 0.160$ & $3.032 \pm 0.522 * *$ & $1.708 \pm 0.385 * *$ \\
\hline TFE-MD & 10 & 140 & $1.252 \pm 0.117$ & $2.735 \pm 0.404 * *$ & $1.163 \pm 0.400 *$ \\
\hline TFE-LD & 10 & 70 & $1.576 \pm 0.420$ & $2.705 \pm 0.518 * *$ & $0.895 \pm 0.191$ \\
\hline
\end{tabular}

Note: compared with the blank group $\Delta P<0.05, \Delta \Delta P<0.01$; compared with the model group $* P<0.05, * * P<0.01$

\subsection{Effects of TFE on serum sex hormones content in climacteric rats}

Compared with the blank group, the level of serum $\mathrm{E}_{2}$ of model group was significantly decreased $(P<0.01), \quad$ LH, FSH levels were significantly increased $(P<0.01)$, explained that incomplete removal of the ovaries induced rat model of menopausal hormone disorders. Compared with the model group, TFE in each dose group and SI group could significantly increase the level of serum E2 $(P<0.01)$, TFE-HD and SI group could significantly decrease the serum LH level, TFE-HD could decrease the levels of FSH $(P<0.01)$. These results are listed in Table 2. 
Table 2 Effect of TFE on the contents of $\mathrm{E}_{2}, \mathrm{FSH}, \mathrm{LH}$ in serum in climacteric model rats $\left(\overline{\mathrm{X}}_{ \pm} \mathrm{s}\right)$

\begin{tabular}{|l|c|c|c|c|c|}
\hline Groups & $\mathrm{n}$ & Dose $(\mathrm{mg} / \mathrm{kg})$ & $\mathrm{E}_{2}(\mathrm{pg} / \mathrm{ml})$ & LH $(\mathrm{IU} / \mathrm{L})$ & FSH $(\mathrm{IU} / \mathrm{L})$ \\
\hline Blank group & 10 & - & $20.19 \pm 1.57$ & $6.127 \pm 0.651$ & $2.830 \pm 0.593$ \\
\hline Model group & 10 & - & $10.96 \pm 1.33^{\triangle \Delta}$ & $7.827 \pm 0.771^{\Delta \Delta}$ & $5.195 \pm 0.991^{\Delta \Delta}$ \\
\hline Gengnian'an capsule group & 10 & 450 & $12.11 \pm 1.14$ & $7.696 \pm 1.299$ & $4.904 \pm 0.817$ \\
\hline SI group & 10 & 85 & $18.55 \pm 1.02^{* *}$ & $6.670 \pm 0.517^{* *}$ & $4.495 \pm 0.663$ \\
\hline TFE-HD & 10 & 210 & $21.23 \pm 2.41^{* *}$ & $6.511 \pm 0.639 * *$ & $4.122 \pm 0.736^{* *}$ \\
\hline TFE-MD & 10 & 140 & $16.58 \pm 2.07^{* *}$ & $7.428 \pm 0.755$ & $4.730 \pm 0.705$ \\
\hline TFE-LD & 10 & 70 & $14.58 \pm 2.37^{* *}$ & $7.565 \pm 0.763$ & $4.613 \pm 0.525$ \\
\hline
\end{tabular}

Note: compared with the blank control group $\Delta P<0.05, \Delta \Delta P<0.01 ;$ compared with the model group $* P<0.05, * * P<0.01$

\subsection{Effects of TFE on IL-2, BGP content in serum of climacteric rats}

Compared with the blank group, the serum IL-2 and BGP levels of model group were significantly decreased $(P<0.01)$, explained that the incomplete removal of the ovaries immunity induced menopause model rats and decreased the bone calcium.
Compared with the model group, Gengnian'an capsule $(P<0.01)$ and TFE-HD $(P<0.05)$ were significantly increase serum IL-2 level; SI group could significantly increase the level of serum BGP $(P<0.05)$, other each group could significantly increase the level of serum BGP $(P<0.05)$. These results are listed in Table 3.

Table 3 Effect of TFE on IL-2, BGP content in serum of climacteric rats $\left(\overline{\mathrm{X}}_{ \pm} \mathrm{s}\right)$

\begin{tabular}{|l|c|c|c|c|}
\hline Groups & $\mathrm{n}$ & Dose $(\mathrm{mg} / \mathrm{kg})$ & $\mathrm{IL}-2(\mathrm{ng} / \mathrm{ml})$ & BGP $(\mu \mathrm{g} / \mathrm{L})$ \\
\hline Blank group & 10 & - & $11.667 \pm 1.570$ & $2.838 \pm 0.311$ \\
\hline Model group & 10 & - & $8.977 \pm 0.529^{\Delta \Delta}$ & $1.685 \pm 0.366^{\Delta \Delta}$ \\
\hline Gengnian'an capsule group & 10 & 450 & $9.901 \pm 1.015^{* *}$ & $2.122 \pm 0.243^{*}$ \\
\hline SI group & 10 & 85 & $9.440 \pm 0.612$ & $2.143 \pm 0.241^{* *}$ \\
\hline TFE-HD & 10 & 210 & $9.367 \pm 0.559 *$ & $2.109 \pm 0.336^{*}$ \\
\hline TFE-MD & 10 & 140 & $9.226 \pm 0.943$ & $2.266 \pm 0.370^{*}$ \\
\hline TFE-LD & 10 & 70 & $9.088 \pm 0.442$ & $2.228 \pm 0.420 *$ \\
\hline
\end{tabular}

Note: compared with the blank control group $\Delta P<0.05, \Delta \Delta P<0.01$; compared with the model group $* P<0.05, * * P<0.01$

\section{DISCUSSION}

Climacteric syndrome is a common disease which frequently occurring, is also a kind of difficult to treat and recurrence disease, it is not only reflected in the gynecological range, but also relates to the department of internal medicine, psychiatric and other fields [6].

Modern medicine thinks that the Pathogenesis of menopausal syndrome is ovarian function gradually decline or loss, estrogen-progesterone levels drop, so that cause the normal hypothalamic-pituitary-ovarian axis balance between disorder. Menopausal women sex hormone levels change significantly, mainly perform as elevated FSH and $\mathrm{LH}, \mathrm{E}_{2}$ decreased. Therefore, this experiment select uterus index, $\mathrm{E}_{2}$, etc as the targets to detect. Modern pharmacological studies have shown that the active constituents of Epimedium and its extract has a wide range of physiological activity. As a traditional Chinese medicine kidney impotence, because of its unique chemical composition and significant biological activity, making it be one of the hot new domestics and international researches.

At present there are three kinds of menopause models both home and abroad, they are natural aging, ovary removal, X-ray irradiation damage the ovaries. Because of ovariectomized animal model time consuming is short and the success rate of the model is high, so this experiment selected ovary removal method making climacteric syndrome animal model[7].

However, there are different between complete removal of the ovaries and human menopausal: not only the synthesis of ovarian estrogen, as well as involved in immune synthesis and secretion of other hormones, human menopausal ovarian function was not completely lost, but completely ovariectomized put these functions completely removed. Therefore, the present study retained some ovaries, ovarian function has not been completely lost, closer to the physiological characteristics of human menopausal syndrome model, and that success rate was high, stable and reliable. 
The experimental results shown that model animal serum $\mathrm{E}_{2}$ levels were significantly reduced, LH, FSH levels were significantly increased, and the level of IL-2 and BGP decreased proved the body's immune level and osteoporosis decreased mainly because of menopause. TFE could increase serum $\mathrm{E}_{2}$ levels menopause animal models, and lower serum FSH and LH levels, indicating that it can regulate hormone levels of menopause animal models; serum BGP rat model of menopause and IL-2 levels significantly enhance the role, indicating TFE could improve immunity due to osteoporosis and menopause, estrogen levels drop due to decline. Through the analysis of experimental results could be seen, TFE could not only improve the environment of climacteric model rats, such as hormone levels, but also had a good curative effect on climacteric diseases caused osteoporosis, decreased immunity and other symptoms.

In summary, TFE have the features of effective, convenient and inexpensive, Epimedium is widely planted in our country, thus to develop new drugs of TFE class, is beneficial to the full use of traditional Chinese medicine resources. In this experiment, examined the effects of TFE on climacteric rat model to investigate the effects of climacteric syndrome and pharmacological mechanism of action, clear pharmacological active ingredients, in order to develop a significant effect of drug treatment of female climacteric syndrome, also establish the experimental basis of developing excellent efficacy of treatment drug. Chinese medicine treatment of climacteric is certainly, broad prospects. In a word, further to study it in depth, will have very important theoretical and practical significance.

\section{ACKNOWLEDGEMENTS}

The research work was supported by Science and Technology Innovation Team of Henan University (2012IRTSTHN011) and the Natural Science Foundation of Henan Province (132300410019).

\section{REFERENCES}

[1] National Pharmacopoeia Committee. 2010. Chinese Pharmacopoeia (a) (M) Beijing: Chinese medicine science and Technology.

[2] Lu, Yue. Xu, Yang \& Cai, Hui.2013. The progress of study on the total Flavonoids of Epimedium. Journal of Shandong University of Traditional Chinese Medicine 37(2): 167.

[3] Zhang, Lei. Wang, Lan. \& Wu, Xia. 2009. The total flavonoids of Herba Epimedii effect on sexual organs in rats with different sex and hormone. Pharmacology and Clinic of Chinese Traditional Medicine 25 (1): 31-33.

[4] Wang, Jing. Zhang, Guilin. \& Ren, Guangyou. 2012. Effects of Epimedin $\mathrm{C}$ and Icariin on uterus of ovariectomized mice. Chinese Journal of clinical pharmacology 28 (3): 193-195.

[5] Huang, Liping. Deng, Minzhen. \& Ye, Murong. 2013. Study on the effects of Gengnianqing tablets on FSH, LH, PRL and E2 in ovariectomized menopausal model rats and the sedation of mice. China Pharmacy24 (15):1366.

[6] Zhang, Ying \& Miao, Mingsan. 2011. Study of commonly used traditional chinese medicine tonifying kidney yang in treating climacteric syndrome. China Journal of Chinese Medicine 9 (26): 1084.

[7] Liu, Ying. Zhao, HongYan. \& Yu, Zheng. 2013. Regulatory effect of nourishing liver and kidney method on the behavior changes of climacteric rats. Chinese journal of basic medicine in Traditional Chinese Medicine 19(4):399. 